\title{
Quantification of happy emotion: Dependence on decisions
}

\author{
Nicoladie D. Tam \\ Department of Biological Sciences, University of North Texas, Denton, TX 76206, USA
}

Email address:

nicoladie.tam@unt.edu

\section{To cite this article:}

Nicoladie D. Tam. Quantification of Happy Emotion: Dependence on Decisions. Psychology and Behavioral Sciences. Vol. 3, No. 2, 2014, pp. 68-74. doi: 10.11648/j.pbs.20140302.16

\begin{abstract}
In the previously proposed theoretical model of emotion, emotion serves as an internal feedback to assess the disparity between the internal prediction and the actual outcomes in the external world, so that congruency between the desirable wants and needs can be met by resolving the emotions. The Emotional-Gain Model predicts that the happy emotional intensity is proportional to the magnitude of the desirable gain signals, while unhappy emotional intensity is proportional to the magnitude loss signals. Using the classical Ultimatum Game (UG) experimental paradigm to elicit self-generated emotions in response to a monetary offer, we want to determine whether the emotional responses are altered in relation to the decision to accept or reject the offer. If so, then does it change the emotional baseline level or the emotional sensitivity? The results showed that the proportionality relationship between emotional intensity and offer-ratios remains the same with respect to the acceptance or rejection decision. The only difference between the decisions is that the baseline level of happiness is shifted by $40 \%$ higher for the decisions to accept the offer, compared to the decisions that rejected the offer. The emotional baseline level is changed without changing the emotional sensitivity. This is quantified by the shift in the $y$-intercept of the emotional stimulus-response function. The happy emotional intensity is shifted upward (toward positive emotion) for those trials that accepted the offer, compared to those who rejected the offer. The slope of the stimulus-response function does not change with respect to the decision, indicating the constancy of the emotional sensitivity. These results validated the hypothesis that happy emotion is inter-related to the decision-making process, such that the decision to accept an offer is related to a shift towards a happier emotion, while the decision to reject an offer is associated with a shift towards an unhappier emotion. This provided the quantitative assessment of how emotion is biased in relation to the decision. The decision to accept an offer is related to a shift to the emotional baseline level rather than a change in the emotional sensitivity — without altering the proportionality relationship between happiness intensity and monetary offer-ratios in UG.
\end{abstract}

Keywords: Emotional Model, Happy, Fairness, Gain, Ultimatum Game, Decision Making, Error Minimization

\section{Introduction}

A theoretical emotional model was proposed previously to describe how emotion is evolved to increase the survivability of an organism by providing the feedback to correct for any discrepancy between the internal predictions and the actual outcomes [1,2]. Using a set of minimal assumptions, it can be shown that the computations in emotional processing involve the assessment of the disparity between the internally generated world model (subjective reality) and the actual real world (objective reality) [3]. This provides a means to detect and correct errors without relying on other external agents. The Emotional-Gain Model predicts that the intensity of emotion is proportional to the gain/loss signals. With a quantitative model available, it provides a set of testable hypotheses to quantify how emotions are processed.

\subsection{Quantification of Emotion in Relation to Decision}

Toward this goal to quantify not only how emotion is processed, but also how emotion is biased using objective measures, the present study addresses how emotion is inter-related to the decision-making process. This study extends the quantified relationship between the emotional intensity and the disparity gain/loss signals described in the companion paper [4], by addressing how this proportionality relationship is related to decisions using the stimulus-response function. 


\subsection{Quantification Using Stimulus-Response Function Derived from the Ultimatum Game Paradigm}

As described in the previous paper [4], a numeric quantitative measure is used to elicit emotions by using the ultimatum game (UG) [5] experimental paradigm without relying on using subjective stimuli, such as facial expression [6] or emotional words [7, 8]. Using this UG paradigm, a stimulus-response function for emotion has been quantified for happy emotion [4] and angry emotion [9]. The emotional stimulus-response function provides a quantifiable measure for revealing how emotions are biased by other factors, such as fairness and decisions. That is, if the perception of fairness and the decision-making process are involved in emotional processing, then the stimulus-response function can be used to quantify how the emotional response is biased by the shifting of the stimulus-response function with respect to decisions.

\subsection{Factors Affecting Emotions}

It has been shown that emotion is an optimization process to minimize the errors in the cost functions [3] with respect to fairness and decisions. The companion paper has quantified that the happy emotion can be biased by the perception of fairness [4], this present study addresses how decision and happy emotion are inter-related quantitatively. It has been shown that decision interacts with emotions, especially in the UG paradigm [10-19]. This paper extends the previous findings by quantifying the specific interactions between emotion and decision, as revealed by the stimulus-response function graphically.

\subsection{Objectives}

The objective of this study is to determine how emotion can be biased by decisions, and how decisions can affect emotion. Although we do not know the causality of this inter-relationship, we want to determine how the emotion is biased in relation to the decision using the UG paradigm. Specifically, if the level of emotion is changed with respect to the acceptance or rejection decision in the UG, we want to quantify whether the decision is correlated with a change in the emotion baseline level or a change in the emotional sensitivity.

\section{Methods}

\subsection{Ultimatum Game Experimental Paradigm}

UG is a widely used experimental paradigm to assess emotions and decision-making responses in neuroscience, psychology, social science, economics and mathematical psychology [5, 11, 18-25]. It is a simple split-the-money game, when an amount of money (such as $\$ 10$ ) is divided between two persons (a proposer and a responder). If the responder accepts the offer, then they both keep the money. If the responder rejects the offer, both lose the money.

Human subjects were recruited to play as the "responder" to accept or reject the monetary offer presented to them on a computer screen. The monetary offer-ratios were randomized, ranging from stingy $\$ 1: \$ 9$ offers to generous $\$ 9: \$ 1$ offers. The experiments were done using the one-shot trial experiment protocol, i.e., the offer was presented once without repeating the same randomized offer-ratios.

Self-reported emotional ratings $(+5$ to -5$)$ were recorded immediately after the participants accepted (or rejected) each offer. The emotional ratings of happy, sad, angry and jealous (using the same +5 to -5 Likert scale [26]) were recorded. The subjects were also asked to rate other variables - whether they won the trial; whether the offer was fair; how important fairness is; how important money is; and how important winning is to them - using the same +5 to -5 scale. These other self-reported ratings served as distracters to reduce the likelihood of the subjects to skew their responses, if they expected which variables the experimenter is addressing. The subjects were not asked explicitly why they opted to accept/reject the offer, similar to other UG studies [9, 13, 19, 24, 25, 27-35], so as not to skew the subject's perception. The experimental protocol was approved by the Institutional Review Board.

\section{Results}

A total of 425 human subjects were included in this study (age ranging from 18 to 80 ; median age $=21$; mean age $=$ 22.3; standard deviation of age $=4.7$ ). The data set included here is the same data set reported in the companion paper [4], except that the data were separated according to the acceptance or rejection decision trials in the analysis. The emotional level with respect to the acceptance and rejection decisions can then be analyzed.

\subsection{Sorting the Emotional Responses by the Decision to Accept or Reject the Monetary Offers}

Fig. 1 shows the self-reported rating of the happiness emotion for the acceptance decision trials to the monetary offer, while Fig. 2 shows the happiness ratings for the rejection decision trials. The emotional responses were sorted according to the ascending order of offer-ratios in the graphs (from the original randomized orders). In order to assess whether the emotional response changed when the subjects accepted vs. rejected the offers, we separate the data analysis according to the acceptance trials (see Fig. 1) or rejection trials (see Fig. 2) for each offer-ratio.

Note that the data points in the graphs are separated according to the acceptance or rejection decision for a specific offer-ratio (independent of whether the same subject accepted or rejected other trials with a different offer-ratio). For instance, the same subject may decide to accept an offer in one trial (to a hyper-fair/hyper-equitable offer), but reject an offer in another trial (to an unfair/inequitable offer). Thus, the response of the same subject may appear in both Fig. 1 and Fig. 2, depending on his/her decision in response to a specific offer for that trial. That is, the subject may be happy 
for one specific offer, but unhappy for another offer. The subject may decide to accept an offer in one trial, but decide to reject another offer in another trial. Note that the subject may not necessarily report happy when he/she accepted the offer, i.e., the subject may accept the offer grudgingly. Thus, by separating the emotional responses based on the

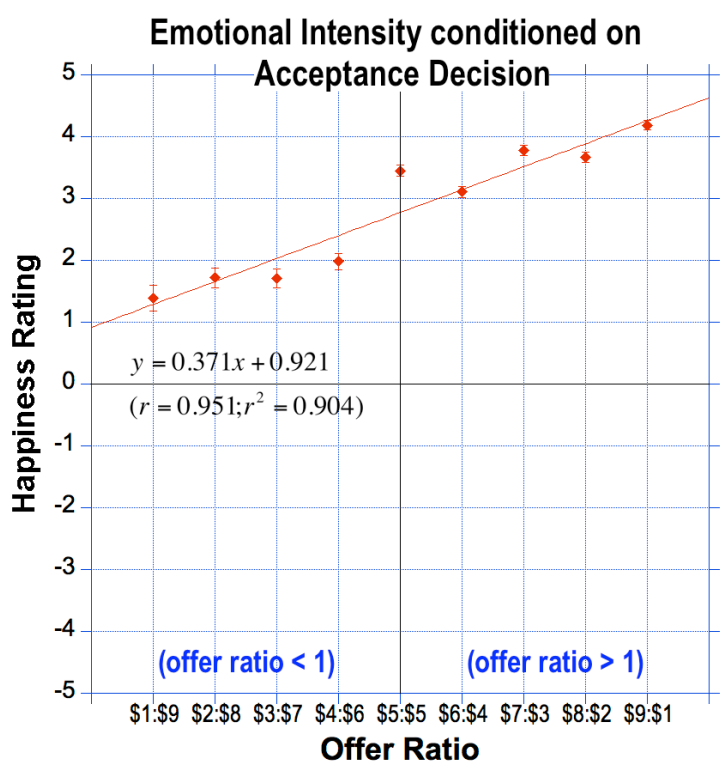

Figure 1. Emotional responses sorted by the condition of acceptance decision for each specific offer-ratio. It shows that the happy emotional intensity is proportional to the offer-ratios. The subjects reported happy when they decide to accept the offers for all offer-ratios (irrespective of whether the offer was equitable or fair). The regression line shows a linear proportional relationship between the emotional intensity and the offer-ratios. Error bars represent standard errors of means (SEMs).

\subsection{Lifting of Baseline Emotional Level for the Acceptance Decision Trials}

Fig. 1 shows the emotional responses for the acceptance trials for the specific offer-ratios. It shows a direct proportional relationship of the self-reported happiness rating to the offer-ratios $\left(r=0.951 ; r^{2}=0.904\right)$, when the subjects accepted the offers. This is similar to the previous findings [4] that the happy emotional intensity is proportional to the offer-ratio, independent of whether they accepts or rejected the offer. The proportionality relationship of the stimulus-response function remains the same, except that the emotional intensity is shifted upward compared to overall responses, without changing the slope (see Fig. 1 [4]) (ANCOVA, $p<0.02 ; 2$-tailed paired-sample $t$-test, $p<0.02$ ).

The human subjects reported being happy to all offer-ratios (even to the most inequitable or unfair offers) when they accepted the offers. The baseline emotion ( $y$-intercept) is at the happy region (positive ratings above the horizontal-axis). There is no minimum offer that they reported unhappy. They were happy to any monetary gain, when they decided to accept the money. This is consistent with the interpretation that they would accept any monetary offers, when they consider the offers as free money. There acceptance or rejection trials, it will allow us to determine whether the subjects were happy (or unhappy), when they accepted (or rejected) a specific offer-ratio. Furthermore, it will allow us to determine which offer-ratio would their emotion switch from happy to unhappy, i.e., identifying the threshold offer-ratio for happiness.

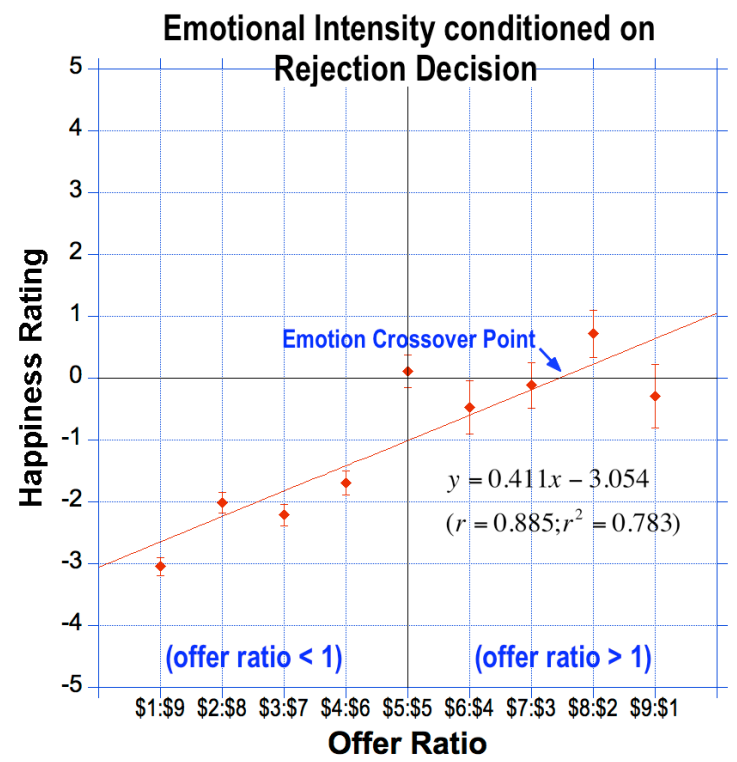

Figure 2. Emotional responses sorted by the condition of rejection decision for each specific offer-ratio. It shows that the happy emotional intensity is also proportional to the offer-ratios, similar to those who accepted the offers in Fig. 1 (except that the emotional level is shifted down, indicating their unhappiness). For rejection decision trials, only the baseline emotional level is shifted compared to the acceptance trials, without changing the slope (i.e., the emotional sensitivity).

were probably no reasons to reject them, irrespective of whether the offer-ratio is equitable or not.

This stimulus-response function quantifies the emotional bias toward happiness when the subjects accepted the offers independent of how much money they were offered. On the other hand, it takes a minimum of an $\$ 2: \$ 8$ offer to be happy for the overall population response (see Fig, 1 in [4]). This indicates a shift toward happier emotion, when they accepted the offer, compared to the overall population.

The baseline emotion ( $y$-intercept) is increased to the happy rating of +0.921 point in the 10 -point scale (above the horizontal-axis) (see regression line: $y=0.371 x+0.921$ in Fig. 1). This shows the elevation of the baseline happiness level to a positive emotion when the subjects accepted the offer, irrespective of whether the offer is fair or not, equitable or not (for all offer-ratios).

\subsection{Reduction of Baseline Emotional Level for the Rejection Decision Trials}

When the subjects rejected the offers (Fig. 2), the happiness ratings were still proportional to the monetary offer-ratio ( $r=0.885 ; r^{2}=0.783$ ), i.e., the proportional relationship remains unchanged. The only difference is that 
the emotional response is shifted down compared to Fig. 1 (ANCOVA, $p<0.00001 ; 2$-tailed paired-sample $t$-test, $p<$ 0.00001). The emotional intensity for those trials that rejected the offers was significantly lower than those trials that accepted the offers. This shows how the emotional bias is related to the decision, which is quantified by the shifting of the stimulus-response function.

The baseline emotion ( $y$-intercept) is reduced to the unhappy region of -3.054 point in the 10-point scale (below the horizontal-axis) (see regression line: $y=0.411 x-3.054$ in Fig. 2). This is a significant reduction of emotional intensity from $+0.921(y$-intercept for the acceptance trials in Fig. 1) to -3.054 ( $y$-intercept for the rejection trials in Fig. 2), i.e., a $-40 \%$ reduction in the baseline emotional intensity. The emotional sensitivity remains essentially unchanged, as revealed by the slopes of the stimulus-response function (comparing Figs. 1 and 2).

\subsection{Shifting of Emotional Crossover Threshold to the Right for the Rejection Decision Trials}

The emotional crossover point (threshold) is shifted to the right at the $\$ 7.5: \$ 2.5$ offer-ratio (Fig. 2) when the subject rejected the offers. That is, it required a minimum offer of a $\$ 7.5: \$ 2.5$ offer to make them happy. They were unhappy below that minimum, suggesting they were probably too greedy to expect a hyper-fair (hyper-equitable) offer to be happy. Thus, this analysis allows us to determine what the threshold emotion is for happiness, as revealed by the crossover point in the emotional stimulus-response function graphically. Shifting of the emotional crossover threshold to the right (for the rejection case) suggests greediness, as it requires a more hyper-equitable amount to make a person happy. On the other hand, shifting of the crossover point to the left (for the acceptance case) suggests leniency, as the person is happy to accept even an inequitable amount of money between the two parties.

\section{Discussion}

The analysis shows that the emotional intensity level is related to the decision to accept or reject the monetary offer. The stimulus-response function quantifies graphically that the happiness level is $40 \%$ higher for the decision trials to accept the offer than the decision to reject the offer. The emotional sensitivity remains unchanged.

\subsection{Quantification of Emotional Baseline in Relation to Decision by the Shifting of the Baseline in the Stimulus-Response Function}

Specifically, the shifting of emotional stimulus-response curve up/down quantifies this emotioinal bias, depending on whether the subjects accepted/rejected the offer. The emotional curve is shifted to the happier emotion by $+40 \%$ in the $y$-intercept (4 points in the 10-point scale) for those who accepted the offer compared to those who rejected the offer (comparing Fig. 1 with Fig. 2). The graphs clearly show that when the subject decided to accept the offers, he/she reported being happy (see Fig. 1). In contrast, if he/she rejected the money, he/she reported unhappy, unless the offer was hyper-fair/hyper-equitable (at least $\$ 7.5: \$ 2.5$ for the emotional crossover threshold) (see Fig. 2).

Note also that the graphs do not necessarily imply any causal relationship between emotions and decision (i.e., whether being happy would make them accept the offer, or accepting the offer would make them happy). The stimulus-response function merely indicates how the emotional intensity is correlated with the decision to accept or reject the offer for a specific offer-ratio trial.

The proportional relationship between emotional intensity and the offer-ratio does not change with the decision. The emotional sensitivity (as represented by the slope of the stimulus-response function) remains the same for both acceptance and rejection trials. The only difference is the shift in the baseline emotional level by $-40 \%$ from happy to unhappy when the decision is acceptance vs. rejection. This suggests that the subject responded similarly for the acceptance or rejection decision, except that the baseline emotional intensity level changes from positive to negative.

\subsection{Quantification of Emotional Threshold in Relation to Decision by Shifting the Happiness Crossover Threshold Left/Right in the Stimulus-Response Function}

The graphs also reveal the bias in happiness threshold, which is not centered on $\$ 5: \$ 5$ fair-share offer (at the axes-origin for neutral emotion), but shifted to either left or right of the graph depending on the subject's subjective bias to fairness [9, 36-38]. The crossover emotional threshold point is shifted to the left when the subject accepted the offer (see Fig. 1), and shifted to the right when the subject rejected it (see Fig. 2). This is consistent with similar shifts in anger threshold crossover point [9], and decision threshold crossover point [39] were reported.

This analysis suggests that the level of happiness is related to the decision, independent of the sense of fairness. If fairness is centered on the absolute equity $\$ 5: \$ 5$ offer-ratio (fair share) in social transactions [18, 19, 27, 28], where offer-ratios $>\$ 5: \$ 5$ is considered as hyper-fair, and offer-ratios $<\$ 5: \$ 5$ is considered as unfair, then the analysis suggests that the happiness level is more related to the decision than the perception of fairness, unless the perception of fairness is also biased. It has been shown that fairness bias occurs in other studies. Human subjects do tolerate a small amount of unfairness by accepting some inequity as fair rather than insisting absolute equity as fair $[9,38]$. The fairness threshold can be shifted, such that an inequitable offer-ratio of $\$ 2: \$ 8$ is still considered as fair instead of $\$ 5: \$ 5[9,38]$. 


\subsection{Quantification of the Constancy in Emotional Sensitivity irrespective of the Decision by Keeping the Same Slope in the Stimulus-Response Function}

This analysis reveals what the emotional bias is in relation to the decision - it only shifts the emotional baseline up (towards happiness) or down (towards unhappiness), but does not alter the emotional sensitivity. This is delineated by the shift in the emotional baseline ( $y$-intercept), but not in the emotional sensitivity (slope).

Nonetheless, independent of whether the subject decided to accept or reject the offer, the proportional relationship between the self-reported happiness perception and the perceived amount of monetary gains compared to losses remains unchanged. The analysis quantified how much their emotional responses were biased (by shifting up/down or left/right) with respect to their decision graphically.

\subsection{Rejecting Monetary Offers without being Irrational}

This shows that rather than assuming the rejection as an irrational decision (by throwing money away) [11, 29, 30], this analysis of emotion showed that the subject's perception is emotionally consistent - without being irrational - by shifting the emotional baseline ( $y$-intercept) downward by $40 \%$ for the rejection decision, and by shifting the emotional crossover point rightward to the hyper-fair/hyper-equitable offer-ratio. It does not alter the proportional relationship between the emotional response and the offer-ratio (i.e., it did not change the slope of the stimulus-response function).

\subsection{Consistency with Other UG Studies}

This is consistent with the findings in other UG studies that rejection decisions are related to unfairness [27, 28], anger and spite $[25,40]$, or altruistic punishment $[39,41]$ rather than being irrational in the decision. This shift to an unhappy emotion is also consistent with other studies, which showed that "maximizers" (who opt for more choices) are unhappier than those "satisfiers" (who opt for less choices) [42]. That is, those who want both money and fairness could not always get everything that they want, whereas those who choose only one, but not the other can get something that they want. Those who choose to want more may end up getting less in the end, as a result. Choosing one (money only) is more likely to result in a smaller disparity than choosing both (money and fairness); thus the emotional level for happiness could be higher for those who accepted the money than those who rejected it.

This is consistent with other studies that the subject's responses can be biased by decisions [18, 19, 43]. Other factors can also bias the response, such as fairness $[11,25$, $27,28,30,32,40]$, negativity bias [44], and satiation effect [45]. Testosterone can also affect the bias by increasing the rejection rate [46] or decreasing the generosity of the offer in UG [34, 47], whereas oxytocin is shown to increase the generosity of the offer in UG [35].

\section{Conclusion}

This study quantifies graphically how emotions are biased in relation to decision by the emotion stimulus-response function. It also quantified that the baseline emotional level is shifted (in the $y$-intercept) from an unhappy region to a happier region, when the subjects accepted instead of rejected the money, without changing the emotional sensitivity (slope). It also showed that the emotional threshold (crossover point for neutral emotion) is also shifted either to the left or right of the equal-share $(\$ 5: \$ 5)$ offer-ratio, depending on the decision to accept or reject, respectively. The decision and fairness perception only shifted (biased) the emotional response up/down and left/right, but they did not alter the proportional relationship between the emotional intensity and the offer-ratios.

\section{Acknowledgements}

I greatly appreciate Ms. Krista Smith for the helpful suggestions, and for proofreading the manuscript.

\section{References}

[1] D. Tam, "EMOTION-I model: A biologically-based theoretical framework for deriving emotional context of sensation in autonomous control systems," Open Cybern Sys J, vol. 1, pp. 28-46, 2007.

[2] D. Tam, "EMOTION-II model: A theoretical framework for happy emotion as a self-assessment measure indicating the degree-of-fit (congruency) between the expectancy in subjective and objective realities in autonomous control systems," Open Cybern Sys J, vol. 1, pp. 47-60, 2007.

[3] D. Tam, "A theoretical model of emotion processing for optimizing the cost function of discrepancy errors between wants and gets," BMC Neuroscience, vol. 10, p. P11, Jul 13 2009.

[4] Psychology and Behavioral Sciences 2014; 3(2): 60-67.

[5] J. von Neumann, O. Morgenstern, and A. Rubinstein, Theory of games and economic behavior. Princeton, NJ: Princeton University Press, 1953.

[6] F. Schneider, R. C. Gur, R. E. Gur, and L. R. Muenz, "Standardized mood induction with happy and sad facial expressions," Psychiatry Res, vol. 51, pp. 19-31, Jan 1994.

[7] I. Laeger, C. Dobel, U. Dannlowski, H. Kugel, D. Grotegerd, J. Kissler, et al., "Amygdala responsiveness to emotional words is modulated by subclinical anxiety and depression," Behav Brain Res, vol. 233, pp. 508-16, Aug 12012.

[8] M. J. van Tol, L. R. Demenescu, N. J. van der Wee, R. Kortekaas, M. A. N. Marjan, J. A. Boer, et al., "Functional magnetic resonance imaging correlates of emotional word encoding and recognition in depression and anxiety disorders," Biol Psychiatry, vol. 71, pp. 593-602, Apr 12012.

[9] D. N. Tam, "Computation in emotional processing: quantitative confirmation of proportionality hypothesis for angry unhappy emotional intensity to perceived loss," Cogn Comput, vol. 3, pp. 394-415, 2011/06/01 2011. 
[10] A. Bechara, "The role of emotion in decision-making: evidence from neurological patients with orbitofrontal damage," Brain Cogn, vol. 55, pp. 30-40, Jun 2004.

[11] C. Civai, C. Corradi-Dell'Acqua, M. Gamer, and R. I. Rumiati, "Are irrational reactions to unfairness truly emotionally-driven? Dissociated behavioural and emotional responses in the Ultimatum Game task," Cognition, vol. 114, pp. 89-95, Jan 2010.

[12] J. D. Greene, L. E. Nystrom, A. D. Engell, J. M. Darley, and J. D. Cohen, "The neural bases of cognitive conflict and control in moral judgment," Neuron, vol. 44, pp. 389-400, Oct 142004.

[13] K. M. Harle and A. G. Sanfey, "Incidental sadness biases social economic decisions in the Ultimatum Game," Emotion, vol. 7, pp. 876-881, Nov 2007.

[14] S. M. McClure, D. I. Laibson, G. Loewenstein, and J. D. Cohen, "Separate neural systems value immediate and delayed monetary rewards," Science, vol. 306, pp. 503-7, Oct 152004.

[15] E. K. Miller and J. D. Cohen, "An integrative theory of prefrontal cortex function," Annu Rev Neurosci, vol. 24, pp. 167-202, 2001.

[16] G. J. Quirk and J. S. Beer, "Prefrontal involvement in the regulation of emotion: convergence of rat and human studies," Curr Opin Neurobiol, vol. 16, pp. 723-7, Dec 2006.

[17] E. T. Rolls, "Brain mechanisms of emotion and decision-making," Int Congress Series, vol. 1291, pp. 3-13, 2006.

[18] A. G. Sanfey, G. Loewenstein, S. M. McClure, and J. D. Cohen, "Neuroeconomics: cross-currents in research on decision-making," Trends Cogn Sci, vol. 10, pp. 108-16, Mar 2006.

[19] A. G. Sanfey, J. K. Rilling, J. A. Aronson, L. E. Nystrom, and J. D. Cohen, "The neural basis of economic decision-making in the Ultimatum Game," Science, vol. 300, pp. 1755-1758, Jun 132003.

[20] J. H. Kagel and A. E. Roth, The handbook of experimental economics: PRINCETON University Press, 1995.

[21] D. A. Braun, P. A. Ortega, and D. M. Wolpert, "Nash equilibria in multi-agent motor interactions," PLoS Comput Biol, vol. 5, p. e1000468, Aug 2009.

[22] K. Sigmund, C. Hauert, and M. A. Nowak, "Reward and punishment," Proc Natl Acad Sci US A, vol. 98, pp. 10757-10762, Sep 112001.

[23] J. K. Rilling, A. G. Sanfey, J. A. Aronson, L. E. Nystrom, and J. D. Cohen, "The neural correlates of theory of mind within interpersonal interactions," Neuroimage, vol. 22, pp. 1694-703, Aug 2004.

[24] P. Smith and A. Silberberg, "Rational maximizing by humans (Homo sapiens) in an ultimatum game," Anim Cogn, vol. 13, pp. 671-7, Jul 2010.

[25] T. Yamagishi, Y. Horita, H. Takagishi, M. Shinada, S. Tanida, and K. S. Cook, "The private rejection of unfair offers and emotional commitment," Proc Natl Acad Sci U S A, vol. 106, pp. 11520-11523, Jul 142009.
[26] S. S. Komorita, "Attitude content, intensity, and the neutral point on a Likert scale," J Soc Psychol, vol. 61, pp. 327-34, Dec 1963.

[27] B. Güroğlu, W. van den Bos, and E. A. Crone, "Fairness considerations: increasing understanding of intentionality during adolescence," J Exp Child Psychol, vol. 104, pp. 398-409, Dec 2009.

[28] B. Güroğlu, W. van den Bos, S. A. Rombouts, and E. A. Crone, "Unfair? It depends: neural correlates of fairness in social context," Soc Cogn Affect Neurosci, vol. 5, pp. 414-423, Dec 2010.

[29] M. Koenigs and D. Tranel, "Irrational economic decision-making after ventromedial prefrontal damage: evidence from the Ultimatum Game," $J$ Neurosci, vol. 27, pp. 951-6, Jan 242007.

[30] M. A. Nowak, K. M. Page, and K. Sigmund, "Fairness versus reason in the ultimatum game," Science, vol. 289, pp. 1773-1775, Sep 82000.

[31] M. M. Pillutla and J. K. Murnighan, "Unfairness, Anger, and Spite: Emotional Rejections of Ultimatum Offers," Org Behav Human Decis Proc, vol. 68, pp. 208-224, 12// 1996.

[32] G. Tabibnia, A. B. Satpute, and M. D. Lieberman, "The sunny side of fairness: preference for fairness activates reward circuitry (and disregarding unfairness activates self-control circuitry)," Psychol Sci, vol. 19, pp. 339-347, Apr 2008.

[33] M. van 't Wout, R. S. Kahn, A. G. Sanfey, and A. Aleman, "Repetitive transcranial magnetic stimulation over the right dorsolateral prefrontal cortex affects strategic decision-making," Neuroreport, vol. 16, pp. 1849-52, Nov 7 2005.

[34] P. J. Zak, R. Kurzban, S. Ahmadi, R. S. Swerdloff, J. Park, L. Efremidze, et al., "Testosterone administration decreases generosity in the ultimatum game," PLoS One, vol. 4, p. e8330, 2009.

[35] P. J. Zak, A. A. Stanton, and S. Ahmadi, "Oxytocin increases generosity in humans," PLoS One, vol. 2, p. e1128, 2007.

[36] M. L. Halko, Y. Hlushchuk, R. Hari, and M. Schurmann, "Competing with peers: mentalizing-related brain activity reflects what is at stake," Neuroimage, vol. 46, pp. 542-8, Jun 2009.

[37] D. Tam, "Variables governing emotion and decision-making: human objectivity underlying its subjective perception," BMC Neuroscience, vol. 11, p. P96, Jul 202010.

[38] D. N. Tam, "Quantification of fairness bias by a Fairness-Equity Model," BMC Neuroscience, vol. 12, p. P327, 2011.

[39] E. Xiao and D. Houser, "Emotion expression in human punishment behavior," Proc Natl Acad Sci U S A, vol. 102, pp. 7398-401, May 172005

[40] M. M. Pillutla and J. K. Murnighan, "Unfairness, anger, and spite: Emotional rejections of ultimatum offers," Organizational Behavior and Human Decision Processes, vol. 68, pp. 208-224, 12// 1996.

[41] E. C. Seip, W. W. van Dijk, and M. Rotteveel, "On hotheads and Dirty Harries: the primacy of anger in altruistic punishment," Ann N Y Acad Sci, vol. 1167, pp. 190-196, Jun 2009. 
[42] I. Dar-Nimrod, C. D. Rawn, D. R. Lehman, and B. Schwartz, "The maximization paradox: The costs of seeking alternatives," Personality and Individual Differences, vol. 46, pp. 631-635, 4// 2009.

[43] M. van't Wout, R. S. Kahn, A. G. Sanfey, and A. Aleman, "Affective state and decision-making in the Ultimatum Game," Exp Brain Res, vol. 169, pp. 564-8, Mar 2006.

[44] C. K. Morewedge, "Negativity bias in attribution of external agency," J Exp Psychol Gen, vol. 138, pp. 535-45, Nov 2009.
[45] E. A. Murray and A. Izquierdo, "Orbitofrontal cortex and amygdala contributions to affect and action in primates," Ann N Y Acad Sci, vol. 1121, pp. 273-96, Dec 2007.

[46] T. C. Burnham, "High-testosterone men reject low ultimatum game offers," Proc Biol Sci, vol. 274, pp. 2327-30, Sep 222007.

[47] C. Eisenegger, M. Naef, R. Snozzi, M. Heinrichs, and E. Fehr, "Prejudice and truth about the effect of testosterone on human bargaining behaviour," Nature, vol. 463, pp. 356-9, Jan 212010. 